\title{
Pulmonary Nocardiosis in Suspected Tuberculosis Patients: A Systematic Review and Meta-Analysis of Cross-Sectional Studies
}

\author{
Susan Mansuri Mehrabadi ${ }^{1}$, Mina Taraghian², Aliyar Pirouzi ${ }^{3}$, Azad Khaledi ${ }^{3 \text {, }}$ \\ $4^{*}$, Alireza Neshani ${ }^{5}$, Somaye Rashki ${ }^{4}$
}

\section{OPEN ACCESS}

Citation: Susan Mansuri Mehrabadi, Mina Taraghian, Aliyar Pirouzi, Azad khaledi, Alireza Neshani, Somaye Rashki. Pulmonary Nocardiosis in Suspected Tuberculosis Patients: A Systematic Review and Meta-Analysis of Cross-Sectional Studies. Ethiop J Health Sci.2020;

30(2):293.doi:http://dx.doi.org/10.4314/ejhs .v30 i2.17

Received: November 5, 2019

Accepted: November 11, 2019

Published: March 1, 2020

Copyright: (O2020 Susan MM., et al.

This is an open access article distributed under the terms of the Creative Commons Attribution License, which permits unrestricted use, distribution, and reproduction in any medium, provided the original author and source are credited. Funding: Nil

Competing Interests: The authors declare that this manuscript was approved by all authors in its form and that no competing interest exists.

Affiliation and Correspondence: ${ }^{1}$ Medical Student, Department of Medicine, Kazeroun Branch, Islamic Azad University, Kazeroun, Iran

${ }^{2}$ Department of Microbiology, School of Basic Sciences, Hamedan Branch, Islamic Azad University, Hamedan, Iran

${ }^{3}$ Cellular and Molecular Gerash Research Center, Gerash University of Medical Sciences, Gerash, Iran ${ }^{4}$ Infectious Diseases Research Center, Department of Microbiology and Immunology, Faculty of Medicine, Kashan University of Medical Sciences, Kashan, Iran

${ }^{5}$ Department of Microbiology and Virology, Faculty of Medicine, Mashhad University of Medical Sciences, Mashhad, Iran

*Email: azadkh99@gmail.com

\section{ABSTRACT}

BACKGROUND: nocardiosis is an opportunistic infectious disease in immunocompromised patients. The most common form of nocardiosis infection in humans is pulmonary nocrdiosis caused by inhaling Nocardia species from the environment. Thus, this study aimed to evaluate the pulmonary nocardiosis in patients with suspected tuberculosis using systematic review and meta-analysis. METHODS: We conducted a systematic search for cross-sectional studies focused on the pulmonary nocardiosis among patients with pulmonary tuberculosis based on the Preferred Reporting Items for Systematic reviews and Meta-analysis (PRISMA) published from January 2001 to October 2019. The search was conducted in MEDLINE/PubMed, Web of Science, Scopus, Cochrane Library, Google Scholar, Science Direct databases, and Iranian databases. Medical subject headings (MeSH) and text words were searched: "pulmonary nocardiosis", “nocardiosis", OR "nocardial infection", "pulmonary nocardial infections/agents", AND "pulmonary tuberculosis", OR "pulmonary TB", AND “Iran". Two of the reviewers enrolled independently articles published in English and Persian languages according to the inclusion and the exclusion criteria. Comprehensive Meta-Analysis software (Version 3.3.070) was used for meta-analysis.

RESULTS: Only 4 studies met the eligibility criteria. The pulmonary nocardiosis prevalence varied from $1.7 \%$ to $6.7 \%$. The combined prevalence of nocardiosis among patients with suspected pulmonary tuberculosis in Iran was $4.8 \%$ (95\% CI: 3-7.3, $Q=5.8$, $Z=12.7)$. No heterogeneity was observed between studies because $I^{2}$ was 48.3. $N$. cyriacigeorgica and $N$. asteroides were reported as the prevalent isolates, respectively.

CONCLUSIONS: This review showed in patients suspected TB when they were negative in all diagnosis laboratory tests, nocardiosis cases which be considered.

KEYWORDS: Nocardia infection, nocardiosis, Pulmonary

Tuberculosis, Pulmonary Nocardioses 


\section{INTRODUCTION}

Nocardiosis is an opportunistic infectious disease in immunocompromised individuals and in people with healthy immune systems is reported as a case (1-4). The nocardiosis spectrum consists of pulmonary, cutaneous, subcutaneous, cerebral, cutaneous lymphatic or systematic diseases (3-5). The most common form of nocardiosis infection in humans is a pulmonary form caused by inhaling Nocardia species from the environment. So far, more than 50 species of Nocardia have been identified. Of which, the important pathogenic species include $N$. brasiliensis, $N$. asteroids complex, $N$. pseudobrasiliensis, $N$. transvalensis, and N. otitidiscaviarum (6). Nocardia is a Grampositive, obligatory aerobic, catalase-positive, nonmotility, and relative acid-fast microorganism. It is slow-growing and in the culture and tissue environment often forms filaments that convert easily to coccus and bacillus forms (7). The main site of Nocardia species is soil, but it is also found in water, sewage and organic residues of plants (8). However, the geographic prevalence of each species may change tremendously over the world, and some are rare (9). These opportunistic bacteria are facultative intracellular, which with suppressing the immune system, are capable of replacing in different parts of the body including the lungs (10). Also, they tend to expand to blood circulation and cause brain abscesses and skin infections; if they are miss-diagnosed and unless timely treated will be fatal (11). Since patients with immunedeficiency, Hematological malignancies, organ transplant recipients, AIDS, prolonged consumption of corticosteroids, chronic alcoholism and diabetes that are deficient in T-cells, are susceptible to pulmonary nocardiosis (12). Some patients with nocardiosis usually have a chronic underlying pulmonary disease and often under Long-term treatment with high doses of corticosteroids (13). Clinical signs and radiological features of pulmonary nocardiosis are similar to that of pulmonary tuberculosis, but its progress is faster than tuberculosis having a period of several months $(14,15)$. Pulmonary lesions caused by pulmonary nocardiosis are predominantly local and have no clinical symptoms, and are associated with abscess and sometimes pneumonia (16-18). Nocardiosis has a worldwide distribution and affects predominantly people of between 20 and 60 years of age (19). The prevalence of the disease in Iran is not known and often has been reported as a case report. In the USA, the annual incidence rate of the disease was reported between 500-1000 cases (16). In most cases, Nocardia infections are not diagnosed quickly because of a lack of specific clinical symptoms $(20,21)$.

Since these organisms are slow to grow, the rapid and accurate diagnosis of them from other organisms for the treatment of severe infections and as well as prevention of brain abscess formation is essential. The use of molecular methods compared to culture have the high speed, accuracy, sensitivity, and specificity in the identification of Nocardia species(22). There is the lack of comprehensive data on the prevalence of pulmonary nocardiosis in Iran. The present study thus aimed to evaluate the pulmonary nocardiosis in suspected tuberculosis patients using systematic review and meta-analysis of cross-sectional studies.

\section{METHODS AND MATERIALS}

Search strategy: We conducted a systematic search for cross-sectional studies focused on the pulmonary nocardiosis among patients with pulmonary tuberculosis based on the Preferred Reporting Items for Systematic reviews and Metaanalysis (PRISMA) published from January 2001 to October 2019. A systematic literature search was conducted in MEDLINE/PubMed, Web of Science, Scopus, Cochrane Library, Google Scholar, and Science Direct databases. Medical subject headings $(\mathrm{MeSH})$ and text words were searched within titles, abstracts, and keywords. The search strategy was as follows: "pulmonary nocardiosis", "nocardiosis", OR "nocardial infection", "pulmonary nocardia infections/agents", AND "pulmonary tuberculosis", OR "pulmonary TB", AND "Iran". We included articles published in English and Persian languages. Two reviewers, A.K and AN, conducted the literature search. The reference sections of retrieved studies were checked to find further relevant studies.

Inclusion and exclusion criteria: All parts of the studies were read to check them against the eligibility criteria. The inclusion criteria were: a) cross-sectional design, and $b$ ) assessing infection of Nocardia and pulmonary TB. Studies published 
before 2001, case reports, abstracts, editorials, literatures reporting inadequate data, case series, congress articles, meeting reports, letters to editors, articles published in languages other than English and Persian, duplicate publications, all review forms were excluded. This peer-review process was performed independently by two reviewers (AK, AN). Finally, discrepancies between two reviewers were resolved by involving a third reviewer.

Quality evaluation: In the assessment process of the qualities of studies, an appraisal tool for CrossSectional Studies (AXIS) was used. According to its guidelines, twenty items were considered for each study. If the associated data was mentioned, a question was scored 'yes'. In case of any doubt or unclear data, a question was marked 'no' or 'can't tell'. A scoring system was used. Based on the number of questions scored 'yes', the quality of studies was classified as 'strong', 'intermediate', or 'weak' (23). Studies with weak quality were removed from the present review.

Data extraction: In the designed forms, data such as the following were listed: author's name, year of publication, location (province or city), sample size $\left(\mathrm{TB}^{+}\right.$), event (pulmonary nocardiosis), and sample type.

Statistical meta-analysis: Comprehensive MetaAnalysis software (Version 3.3.070) was used for meta-analysis. The statistical significance level was considered as $P<0.05$. The fixed-effects model was used to calculate total effects. Cochran's Q and I square $\left(I^{2}\right)$ tests were used for assessing sources of heterogeneity among studies. Publication bias was checked by Egger's regression asymmetry test.

\section{RESULTS}

Study selection: In total, 234 relevant articles were recognized via the initial literature search (Figure1). About 111 records were excluded due to duplications. Fifty-six studies were removed due to the irrelevant titles. Then, abstracts 67 remained records were screened. It resulted in the exclusion of 13 irrelevant topics. After that, 37 records were excluded because they were case reports. Seventeen full-text literatures were assessed against eligibility criteria which led to the exclusion of 13 other studies that did not satisfy the criteria. Finally, 4 studies were included in the current review (Meta-analysis).

Characteristics of the included studies: All the 4 included cross-sectional studies that had been published between January 2001 to October 2019. Overall, 476 TB suspected patients were enrolled as total sample size. The pulmonary nocardiosis prevalence varied from $1.7 \%$ to $6.7 \%$. Phenotypic methods (i.e. AFB analysis-Direct MicroscopyCulture, Gram staining, and Ziehl-Neelsen technique) and biochemical tests had been used to detect candidiasis in all the studies. The detailed characteristics of the included studies are listed in Table 1.

Table 1: Characteristics of selected studies in this systematic review and meta-analysis

\begin{tabular}{lcccccc}
\hline Study & $\begin{array}{c}\text { Time of } \\
\text { study }\end{array}$ & $\begin{array}{c}\text { Publication } \\
\text { (years) }\end{array}$ & $\begin{array}{c}\text { Sample } \\
\text { size } \\
\text { (suspected } \\
\text { TB) }\end{array}$ & Location & $\begin{array}{c}\text { Samples } \\
\text { type }\end{array}$ & $\begin{array}{c}\text { Pulmonary } \\
\text { nocardiosis } \\
\text { (N, \%) }\end{array}$ \\
\hline $\begin{array}{l}\text { Zaker } \\
\text { Bostanabad(37) }\end{array}$ & $2011-13$ & 2013 & 90 & $\begin{array}{c}\text { Khuzestan- } \\
\text { Tehran }\end{array}$ & $\begin{array}{c}\text { BAL- } \\
\text { Sputum, }\end{array}$ \\
Ekrami(28) & $2011-12$ & 2014 & 32 & Ahvaz & Sputum & $2(6.7)$ \\
Family(38) & $2011-12$ & 2014 & 116 & Tehran & BAL & $7(6)$ \\
Fatahi(39) & $2011-13$ & 2015 & 238 & Tehran & Sputum & $4(1.7)$ \\
\hline
\end{tabular}

Neg: Negative

DOI: http://dx.doi.org/10.4314/ejhs.v30i2.17 


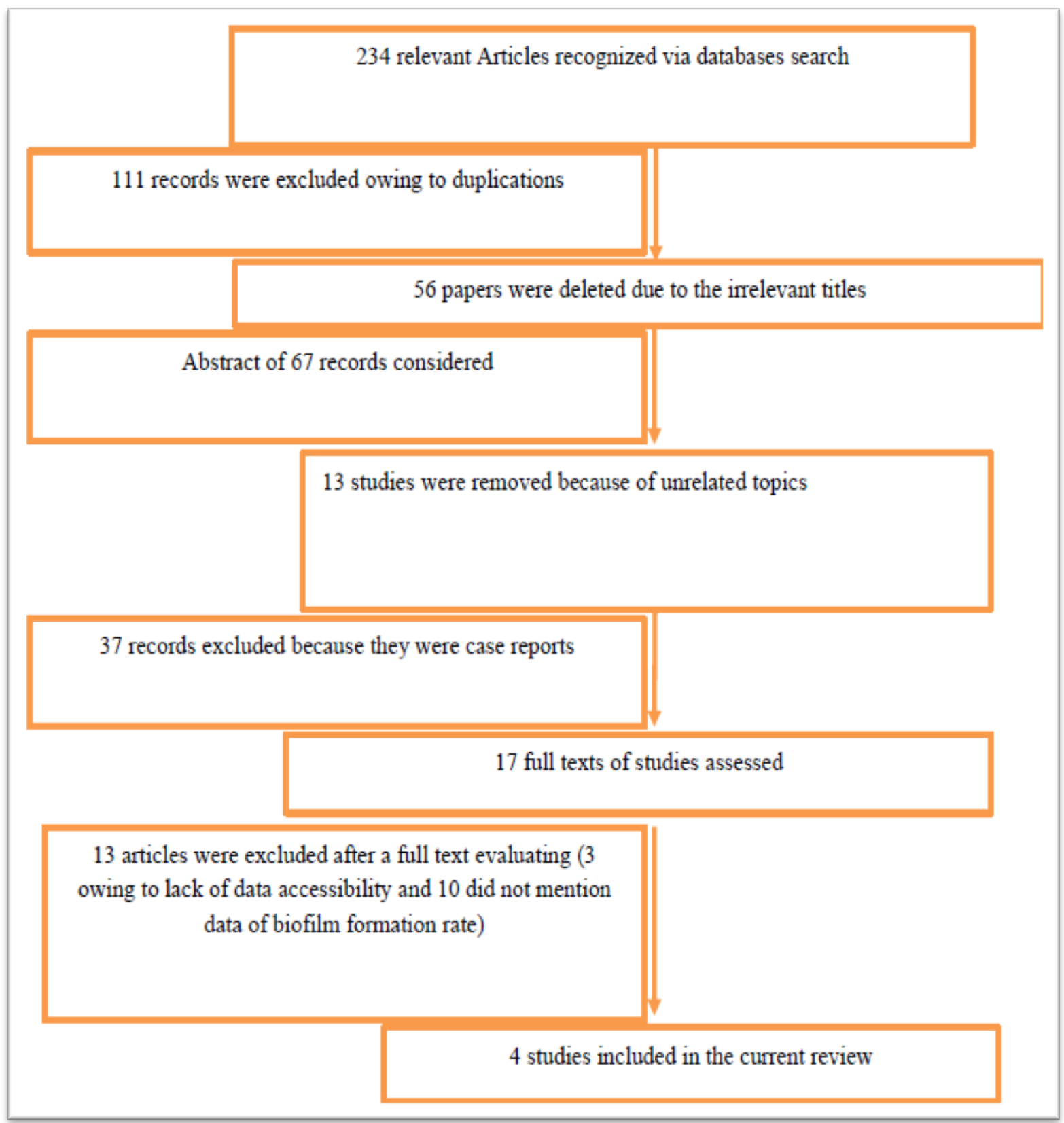

Figure 1: Flow diagram of inclusion process of selected studies

Overall effects: The combined prevalence of nocardiosis among patients with suspected pulmonary tuberculosis in Iran was $4.8 \%$ (95\% CI: 3-7.3, $\mathrm{Q}=5.8, \mathrm{Z}=12.7$ ) (Table 2, Figure 2). No publication bias was detected by the Funnel plot (Figure 3), although Egger's regression test showed the bias $(\mathrm{p}=0.77)$. In addition, no heterogeneity was observed between studies; $\mathrm{I}^{2}$ was 48.3 . Among 4 studies, only 2 reported the Nocardai spp., as Zaker Bostanabad et al. reported 4 and 2 isolates of $N$. asteroides and $N$. wallacei, respectively. Also, Family et al. reported 6 and 1 isolates of $\mathrm{N}$. cyriacigeorgica and N. otitidiscaviarum, respectively. 
Table 2: The overall effect of the prevalence of pulmonary nocardiasis among patients with suspected pulmonary tuberculosis.

\begin{tabular}{|c|c|c|c|c|c|c|c|c|c|}
\hline \multirow{2}{*}{$\begin{array}{l}\text { Overall } \\
\text { effects }\end{array}$} & \multirow{2}{*}{$\begin{array}{l}\text { Number } \\
\text { of studies }\end{array}$} & \multicolumn{3}{|c|}{ Heterogeneity test } & \multicolumn{2}{|c|}{ Egger's test } & \multicolumn{3}{|c|}{ Random model } \\
\hline & & $\begin{array}{l}\text { Prevalence } \\
(95 \% \text { CI) (\%) }\end{array}$ & $\bar{Z}$ & $\mathrm{P}$ & Q & $\mathrm{P}$ & $\mathrm{I}^{2}$ & $\mathrm{~T}$ & $\mathrm{P}$ \\
\hline nocardiosis & 4 & $4.8 \%(3-7.3)$ & 12.7 & 0.121 & 5.8 & 0.77 & 48.3 & 0.32 & 0.00 \\
\hline
\end{tabular}

Figure 2: The Forest plot of the meta-analysis of the prevalence of pulmonary nocardiasis among patients with suspected pulmonary tuberculosis

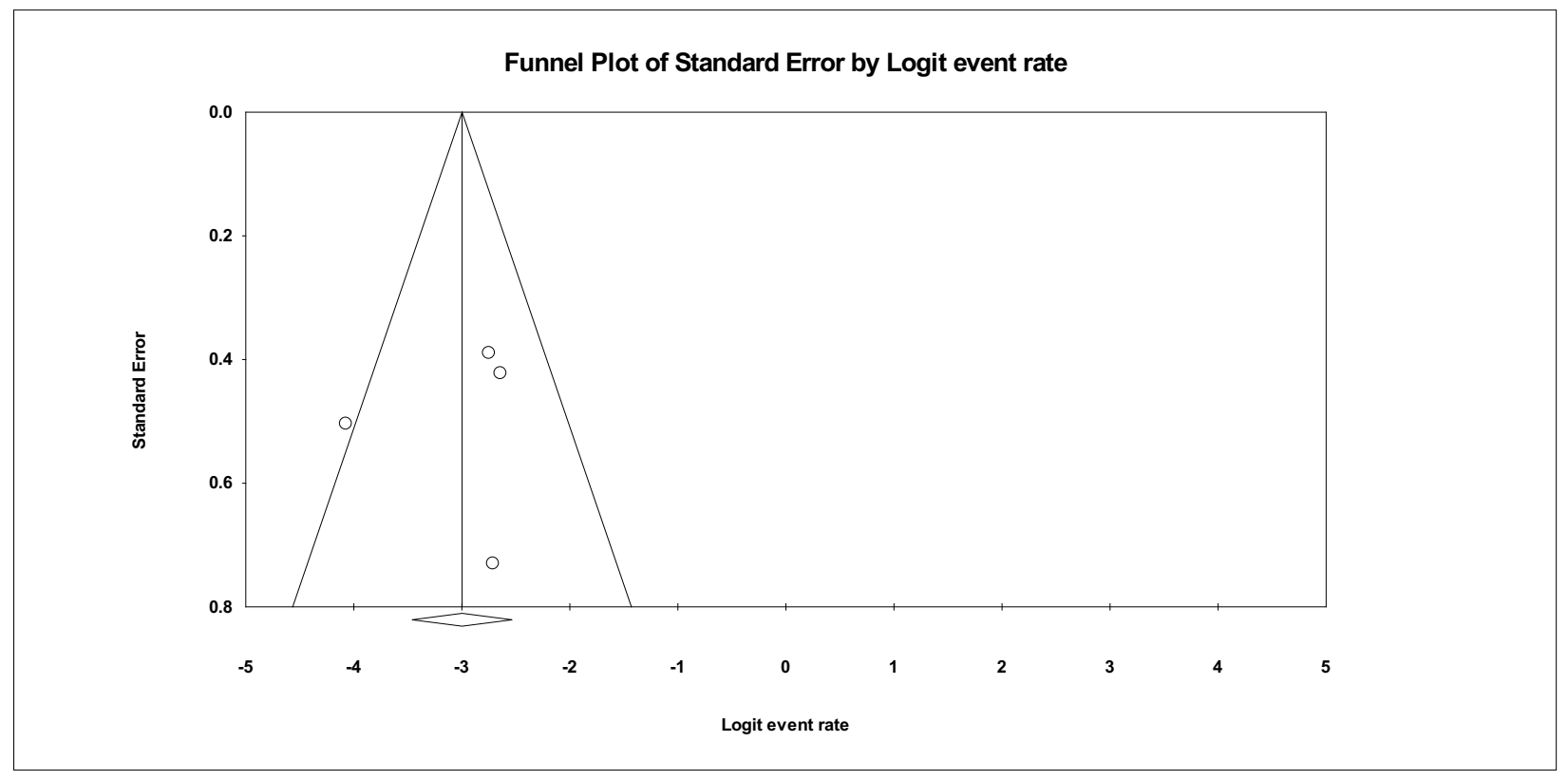

Figure 3: Funnel plot of meta-analysis on the prevalence of pulmonary nocardiasis in patients with suspected pulmonary tuberculosis

\section{DISCUSSION}

In the present systematic review and meta-analysis, we included the studies which reported Nocardia spp. in patient groups who suffered from TB or patients suspected TB. We observed Nocardia in both groups. The noticeable point is that in suspected TB patients with negativity in all TB diagnosis laboratory tests, cases of nocardiosis were confirmed. In some cases, they may be mistaken for tuberculosis due to the lack of specific symptoms (24). Therefore, patients with clinical symptoms of tuberculosis that do not recover Mycobacterium tuberculosis may have other pathogens such as fungi and Nocardia species (25).
Similarly, several patients identified as smearnegative for pulmonary Tuberculosis will have nocardiosis as reported by Rasheed et.al (26).

Molecular techniques are needed to identify this microorganism due to the similarities between nocardiosis and Tuberculosis manifestations (27). In the present review, it was found that, in one study conducted by Ekrami and et al (28), the samples belonged to admitted HIV-infected patients. The samples were studied using phenotypic (Gram and Ziehl-Nelsen staining, culture) and molecular methods (PCR). They reported that two HIV infected patients were positive for Nocardia spp. when PCR used for 
diagnosis of pulmonary tuberculosis. In accordance with their results, Alnaum et al. reported the prevalence of $3-4 \%$ of pulmonary nocardiosis in HIV-infected patients with pulmonary tuberculosis (29). Of course, others reported more co-infection of Nocardia with pulmonary tuberculosis (30).

In patients who do not respond to the usual anti-bacterial or anti-TB treatment, there will be a possibility of nocardiosis (29,31). Accurate identification of Nocardia species has become increasingly important because optimal therapeutic strategies depend on rapid and accurate identification of Nocardia spp. Molecular methods for identification (such as PCR, RFLP, and 16SrRNA sequencing), are time-saving than routine phenotypic methods (9).

Our findings reported the combined prevalence of nocardiosis among patients with suspected pulmonary tuberculosis $4.8 \%$. Prevalence of pulmonary nocardiosis varied from $1.7 \%$ to $6.7 \%$.

This difference in the prevalence of Nocardia in studies included in the current review from Iran possibly can be attributed to geographical location, the methods used for detection (phenotypic and molecular methods), and also patient groups (patients suspected of TB or HIV patients who suffered from TB) (32). Nocardiosis is a global concern, as Nocardia infections are not rare with an annual prevalence rate between 500-1,000 cases, $85 \%$ of which are pulmonary or systemic infections (33).

Also, the studies included in this review reported the N. cyriacigeorgica and $N$. asteroides as the prevalent isolates, respectively. Inconsistent with our findings, $N$. asteroids is the most causative agent in most pulmonary nocardiosis cases in the USA and Europian countries (33).

The outcomes of pulmonary nocardiosis are very bad. The mortality rate resulted from pulmonary nocardiosis is between $30-35 \%$ and increased to $64 \%$ in the disseminated form $(13,34$ 36).

We understood the importance and outcome of pulmonary nocardiosis in suspected pulmonary TB patients and its misdiagnosis with $\mathrm{TB}$ since the manifestations are similar. Thus, it is important to establish molecular techniques with high sensitivity and specificity alongside conventional phenotypic methods to improve the speed of diagnosis of nocardiosis.

In the present systematic review and metaanalysis, we included studies that reported Nocardia spp. in patient groups who suffered from TB or patients suspected TB. We observed in both groups that Nocardia was seen. The noticeable point was that in patients suspected of $\mathrm{TB}$, where all tests used for detection of $\mathrm{TB}$, were negative while cases of nocardiosis were confirmed which is considered. Therefore, implanting molecular methods alongside conventional phenotypic methods to improve the speed of diagnosis of nocardiosis is necessary.

\section{ACKNOWLEDGMENTS}

We would like to thank our friends for helping in the search process.

\section{REFERENCES}

1. Fatahi Bafghi M, Saeed Eshraghi S, Heidarieh P, Habibnia S, Nasab MR. Nocardiosis in immune disorder disease. Malaysian Journal of Medical Sciences. 2014;21(1):75-6.

2. Makwana G, Sinha M, Jain V, Jaiswal A. Pulmonary nocardiosis- a rare case of pulmonary nocardiosis in an immunocompetent patient. Journal of the Indian Medical Association. 2013;111(3):194.

3. Forbes B, Sahm D, Weissfeld A. Nocardia, Streptomyces, Rhodococcus, and similar organisms. Bailey \& Scott's Diagnostic Microbiology: A Text book of Microbiology 12th ed MOSBY, Elsevier. 2007:311-22.

4. Beaman BL, Beaman L. Nocardia species: host-parasite relationships. Clinical microbiology reviews. 1994;7(2):213-64.

5. Iketani Y, Hata Y, Yamamoto N, Oguri T. Case of the abscess type cutaneous nocardiosis. Medical mycology journal. 2014;55(1):J19-23.

6. Borriello SP, Murray PR, Funke G. Topley and Wilson's Microbiology and Microbial Infections: Bacteriology: Wiley Online Library; 2005.

7. Mordarski M, Williams ST, Goodfellow M. The biology of the actinomycetes. Academic Press, 1983;24: 224-232. 
8. Tuomela M, Vikman M, Hatakka A, Itävaara M. Biodegradation of lignin in a compost environment: a review. Bioresource technology. 2000;72(2):169-83.

9. Saubolle MA, Sussland D. Nocardiosis: review of clinical and laboratory experience. Journal of clinical microbiology. 2003;41(10):4497-501.

10. Mahon CR, Lehman DC, Manuselis G. Textbook of diagnostic microbiology-e-book: Elsevier Health Sciences; 2018;16(3):556-581.

11. Roth A, Andrees S, Kroppenstedt RM, Harmsen D, Mauch H. Phylogeny of the genus Nocardia based on reassessed 16S rRNA gene sequences reveals underspeciation and division of strains classified as Nocardia asteroides into three established species and two unnamed taxons. Journal of clinical microbiology. 2003;41(2):851-6.

12. Gaude G, Hemashettar B, Bagga A, Chatterji R. Clinical profile of pulmonary nocardiosis. The Indian journal of chest diseases \& allied sciences. 1999;41(3):153-7.

13. Burgert SJ. Nocardiosis: a clinical review. Infectious Diseases in Clinical Practice. 1999;8(1):27-32.

14. Khaledi A, Bahador A, Esmaeili D, Tafazoli A, Ghazvini K, Mansury D. Prevalence of nontuberculous mycobacteria isolated from environmental samples in Iran: A metaanalysis. Journal of research in medical sciences: the official journal of Isfahan University of Medical Sciences. 2016;21:2032011.

15. Ashna H, Kaffash A, Khaledi A, Ghazvini K. Mutations of rpob Gene Associated with Rifampin Resistance among Mycobacterium Tuberculosis Isolated in Tuberculosis Regional Reference Laboratory in Northeast of Iran during 2015-2016. Ethiopian journal of health sciences. 2018;28(3):299-304.

16. Darazam IA, Shamaei M, Mobarhan M, Ghasemi S, Tabarsi P, Motavasseli M, et al. Nocardiosis: risk factors, clinical characteristics and outcome. Iranian Red Crescent Medical Journal. 2013;15(5):436.

17. Amiri MJ, Karami P, Chichaklu AH, Jangan EH, Amiri MJ, Owrang M, et al. Identification and Isolation of Mycobacterium tuberculosis from Iranian Patients with Recurrent TB using Different Staining Methods. J Res Med Dent Sci. 2018;6(2):409-14.

18. Amiri MRJ, Siami R, Khaledi A. Tuberculosis Status and Coinfection of Pulmonary Fungal Infections in Patients Referred to Reference Laboratory of Health Centers Ghaemshahr City during 2007-2017. Ethiopian journal of health sciences. 2018;28(6):93-101.

19. Corti ME, Fioti MEV. Nocardiosis: a review. International journal of infectious Diseases. 2003;7(4):243-50.

20. Couble A, Rodríguez-Nava V, de Montclos MP, Boiron P, Laurent F. Direct detection of Nocardia spp. in clinical samples by a rapid molecular method. Journal of clinical microbiology. 2005;43(4):1921-4.

21. Eshraghi SS, Talebi M, Namaki S, Mirshafiey A. Nocardia. Journal of Chinese Clinical Medicine. 2009;4:717-724.

22. Wilson RW, Steingrube VA, Brown BA, Wallace RJ. Clinical application of PCRrestriction enzyme pattern analysis for rapid identification of aerobic actinomycete isolates. Journal of clinical microbiology. 1998;36(1):148-52.

23. Downes MJ, Brennan ML, Williams HC, Dean RS. Development of a critical appraisal tool to assess the quality of cross-sectional studies (AXIS). BMJ open. 2016;6(12):e011458.

24. Peleg AY, Husain S, Qureshi ZA, Silveira FP, Sarumi M, Shutt KA, et al. Risk factors, clinical characteristics, and outcome of Nocardia infection in organ transplant recipients: a matched case-control study. Clinical Infectious Diseases. 2007;44(10):1307-14.

25. Schlaberg R, Huard RC, Della-Latta P. Nocardia cyriacigeorgica, an emerging pathogen in the United States. Journal of clinical microbiology. 2008;46(1):265-73.

26. Rasheed M, Belay G. Nocardiosis in HIV seropositive clinically suspected pulmonary tuberculosis patients. Tropical doctor. 2008;38(1):34-5. 
27. Brown-Elliott BA, Brown JM, Conville PS, Wallace RJ. Clinical and laboratory features of the Nocardia spp. based on current molecular taxonomy. Clinical microbiology reviews. 2006;19(2):259-82.

28. Ekrami A, Khosravi AD, Zadeh ARS, Hashemzadeh M. Nocardia co-infection in patients with pulmonary tuberculosis. Jundishapur journal of microbiology. 2014;7(12):1-4.

29. Alnaum HM, Elhassan MM, Mustafa FY, Hamid ME. Prevalence of Nocardia species among HIV-positive patients with suspected tuberculosis. Tropical doctor. 2011;41(4):2246.

30. Pintado V, Gómez-Mampaso E, Cobo J, Quereda C, Meseguer M, Fortún J, et al. Nocardial infection in patients infected with the human immunodeficiency virus. Clinical microbiology and infection. 2003;9(7):716-20.

31. Khaledi A, Khademi F, Esmaeili D, Esmaeili $\mathrm{S}$, Rostami $\mathrm{H}$. The role of $\mathrm{HPaA}$ protein as candidate vaccine against. Helicobacter pylori. 2016:235-7.

32. Imai K, Koibuchi $T$, Kikuchi $T$, Koga $M$, Nakamura H, Miura $\mathrm{T}$, et al. Pulmonary nocardiosis caused by Nocardia exalbida complicating Pneumocystis pneumonia in an HIV-infected patient. Journal of Infection and Chemotherapy. 2011;17(4):547-51.

33. Beaman B, Burnside J, Edwards B, Causey W. Nocardial infections in the United States,
1972-1974. Journal of Infectious Diseases. 1976;134(3):286-9.

34. Martinez Tomas R, Menendez Villanueva R, Reyes Calzada S, Santos Durantez M, Valles Tarazona JM, Modesto Alapont $\mathrm{M}$, et al. Pulmonary nocardiosis: risk factors and outcomes. Respirology. 2007;12(3):394-400.

35. Mari B, Montón C, Mariscal D, Luján M, Sala M, Domingo C. Pulmonary nocardiosis: clinical experience in ten cases. Respiration. 2001;68(4):382-8.

36. Hui $\mathrm{C}, \mathrm{Au} \mathrm{V}$, Rowland $\mathrm{K}$, Slavotinek J, Gordon D. Pulmonary nocardiosis re-visited: experience of 35 patients at diagnosis. Respiratory medicine. 2003;97(6):709-17.

37. Zaker Bostanabad HS. Isolation of nocardia from clinical specimens of patients with suspected tuberculosis and their identification. New Cellular and Molecular Biotechnology Journal. 2014;4(13):19-23.

38. AB Family RK, R Mirnejad. Identification of Nocardiosis Factors in Bronchial Wash (BAL) Samples of Tuberculosis Patients Referred to Tehran Hospitals using PCR. Yakhteh. 2016;16(4):126-134

39. Bafghi MF, Heidarieh P, Soori T, Saber S, Meysamie A, Gheitoli K, et al. Nocardia isolation from clinical samples with the paraffin baiting technique. Germs. 2015;5(1):12. 